Bull. Korean Math. Soc. 52 (2015), No. 1, pp. 323-333

http://dx.doi.org/10.4134/BKMS.2015.52.1.323

\title{
FINITE SETS WITH FAKE OBSERVABLE CARDINALITY
}

\author{
Alfonso Artigue
}

\begin{abstract}
Let $X$ be a compact metric space and let $|A|$ denote the cardinality of a set $A$. We prove that if $f: X \rightarrow X$ is a homeomorphism and $|X|=\infty$, then for all $\delta>0$ there is $A \subset X$ such that $|A|=4$ and for all $k \in \mathbb{Z}$ there are $x, y \in f^{k}(A), x \neq y$, such that $\operatorname{dist}(x, y)<\delta$. An observer that can only distinguish two points if their distance is grater than $\delta$, for sure will say that $A$ has at most 3 points even knowing every iterate of $A$ and that $f$ is a homeomorphism. We show that for hyperexpansive homeomorphisms the same $\delta$-observer will not fail about the cardinality of $A$ if we start with $|A|=3$ instead of 4 . Generalizations of this problem are considered via what we call $(m, n)$-expansiveness.
\end{abstract}

\section{Introduction}

Since 1950, when Utz [16] initiated the study of expansive homeomorphism, several variations of the definition appeared in the literature. Let us recall that a homeomorphism $f: X \rightarrow X$ of a compact metric space $(X$, dist) is expansive if there is an expansive constant $\delta>0$ such that if $x \neq y$, then $\operatorname{dist}\left(f^{k}(x), f^{k}(y)\right)>\delta$ for some $k \in \mathbb{Z}$. Some variations of this definition are weaker, as for example continuum-wise expansiveness [6] and $N$-expansiveness [9] (see also [3, 8, 13]). A branch of research in topological dynamics investigates the possibility of extending known results for expansive homeomorphisms to these versions. See for example $[2,5,10,12,14]$.

Other related definitions are stronger than expansiveness as for example positive expansiveness [15] and hyper-expansiveness [1]. Both definitions are so strong that their examples are almost trivial. It is known [15] that if a compact metric space admits a positive expansive homeomorphism, then the space has only a finite number of points. Recall that $f: X \rightarrow X$ is positive expansive if there is $\delta>0$ such that if $x \neq y$, then $\operatorname{dist}\left(f^{k}(x), f^{k}(y)\right)>\delta$ for some $k \geq 0$. Therefore, we have that if the compact metric space $X$ is not a finite set, then for every homeomorphism $f: X \rightarrow X$ and for all $\delta>0$ there are $x \neq y$ such that $\operatorname{dist}\left(f^{k}(x), f^{k}(y)\right)<\delta$ for all $k \geq 0$. This is a very general result about the dynamics of homeomorphisms of compact metric spaces.

Received February 18, 2014.

2010 Mathematics Subject Classification. 54H20, 37B05.

Key words and phrases. topological dynamics, expansive homeomorphisms.

(C)2015 Korean Mathematical Society 
Another example of this phenomenon is given in [1], where it is proved that no uncountable compact metric space admits a hyper-expansive homeomorphism (see Definition 3). Therefore, if $X$ is an uncountable compact metric space, as for example a compact manifold, then for every homeomorphism $f: X \rightarrow X$ and for all $\delta>0$ there are two compact subsets $A, B \subset X, A \neq B$, such that $\operatorname{dist}_{H}\left(f^{k}(A), f^{k}(B)\right)<\delta$ for all $k \in \mathbb{Z}$. The distance $\operatorname{dist}_{H}$ is called Hausdorff metric and its definition is recalled in equation (3) below.

According to Lewowicz [7] we can explain the meaning of expansiveness as follows. Let us say that a $\delta$-observer is someone that cannot distinguish two points if their distance is smaller than $\delta$. If $\operatorname{dist}(x, y)<\delta$ a $\delta$-observer will not be able to say that the set $A=\{x, y\}$ has two points. But if the homeomorphism is expansive, with expansive constant greater than $\delta$, and if the $\delta$-observer knows all of the iterates $f^{k}(A)$ with $k \in \mathbb{Z}$, then he will find that $A$ contains two different points, because if $\operatorname{dist}\left(f^{k}(x), f^{k}(y)\right)>\delta$, then he will see two points in $f^{k}(A)$. Let us be more precise.

Definition 1. For $\delta \geq 0$, a set $A \subset X$ is $\delta$-separated if for all $x \neq y, x, y \in A$, it holds that $\operatorname{dist}(x, y)>\delta$. The $\delta$-cardinality of a set $A$ is

$$
|A|_{\delta}=\sup \{|B|: B \subset A \text { and } B \text { is } \delta \text {-separated }\},
$$

where $|B|$ denotes the cardinality of the set $B$.

Notice that the $\delta$-cardinality is always finite because $X$ is compact. The $\delta$-cardinality of a set represents the maximum number of different points that a $\delta$-observer can identify in the set.

In this paper we introduce a series of definitions, some weaker and other stronger than expansiveness, extending the notion of $N$-expansiveness of [9]. Let us recall that given $N \geq 1$, a homeomorphism is $N$-expansive if there is $\delta>0$ such that if $\operatorname{diam}\left(f^{k}(A)\right)<\delta$ for all $k \in \mathbb{Z}$, then $|A| \leq N$. In terms of our $\delta$-observer we can say that $f$ is $N$-expansive if there is $\delta>0$ such that if $|A|=N+1$, a $\delta$-observer will be able to say that $A$ has at least two points given that he knows all of the iterates $f^{k}(A)$ for $k \in \mathbb{Z}$, i.e., $\left|f^{k}(A)\right|_{\delta}>1$ for some $k \in \mathbb{Z}$. Let us introduce our main definition.

Definition 2. Given integer numbers $m>n \geq 1$ we say that $f: X \rightarrow X$ is $(m, n)$-expansive if there is $\delta>0$ such that if $|A|=m$, then there is $k \in \mathbb{Z}$ such that $\left|f^{k}(A)\right|_{\delta}>n$.

The first problem under study is the classification of these definitions. We prove that $(m, n)$-expansiveness implies $N$-expansiveness if $m \leq(N+1) n$. In particular, if $m \leq 2 n$, then $(m, n)$-expansiveness implies expansiveness. These results are stated in Corollary 1.7. It is known that even on surfaces, $N$ expansiveness does not imply expansiveness for $N \geq 2$, see [2]. Here we show that $(m, n)$-expansiveness does not imply expansiveness if $n \geq 2$. For example, Anosov diffeomorphisms are known to be expansive and a consequence of Theorem 5.1 is that Anosov diffeomorphisms are not $(m, n)$-expansive for all $n \geq 2$. 
It is a fundamental problem in dynamical systems to determine which spaces admit expansive homeomorphisms (or Anosov diffeomorphisms). In this paper we prove that no Peano continuum admits a $(m, n)$-expansive homeomorphism if $2 m \geq 3 n$, see Theorem 3.2. We also show that if $X$ admits a $(n+1, n)$-expansive homeomorphism with $n \geq 3$, then $X$ is a finite set. Examples of $(3,2)$-expansive homeomorphisms are given on countable spaces (hyper-expansive homeomorphisms), see Theorem 4.1.

The article is organized as follows. In Section 1 we prove basic properties of $(m, n)$-expansive homeomorphisms. In Section 2 we prove the first statement of the abstract, i.e., no infinite compact metric space admits a $(4,3)$-expansive homeomorphism. In Section 3 we show that no Peano continuum admits a $(m, n)$-expansive homeomorphism if $2 m \geq 3 n$. In Section 4 we show that hyper-expansive homeomorphisms are $(3,2)$-expansive. Such homeomorphisms are defined on compact metric spaces with a countable number of points. In Section 5 we prove that a homeomorphism with the shadowing property and with two points $x, y$ satisfying

$$
0=\liminf _{k \rightarrow \infty} \operatorname{dist}\left(f^{k}(x), f^{k}(y)\right)<\limsup _{k \rightarrow \infty} \operatorname{dist}\left(f^{k}(x), f^{k}(y)\right)
$$

cannot be $(m, 2)$-expansive if $m>2$.

I would like to thank M. J. Pacifico and J. L. Vieitez for their kind comments and suggestions on preliminary versions of the article.

\section{Separating finite sets}

Let $(X$, dist $)$ be a compact metric space and consider a homeomorphism $f: X \rightarrow X$. Let us recall that for integer numbers $m>n \geq 1$ a homeomorphism $f$ is $(m, n)$-expansive if there is $\delta>0$ such that if $|A|=m$, then there is $k \in \mathbb{Z}$ such that $\left|f^{k}(A)\right|_{\delta}>n$. In this case we say that $\delta$ is a $(m, n)$-expansive constant. The idea of $(m, n)$-expansiveness is that our $\delta$-observer will find more than $n$ points in every set of $m$ points if he knows all of its iterates.

Remark 1.1. From the definitions it follows that a homeomorphisms is $(N+$ $1,1)$-expansive if and only if it is $N$-expansive in the sense of [9]. In particular, $(2,1)$-expansiveness is equivalent with expansiveness.

Remark 1.2. Notice that if $X$ is a finite set, then every homeomorphism of $X$ is $(m, n)$-expansive.

Proposition 1.3. If $n^{\prime} \leq n$ and $m-n \leq m^{\prime}-n^{\prime}$, then $(m, n)$-expansive implies $\left(m^{\prime}, n^{\prime}\right)$-expansive with the same expansive constant.

Proof. The case $|X|<\infty$ is trivial, so, let us assume that $|X|=\infty$. Consider $\delta>0$ as a $(m, n)$-expansive constant. Given a set $A$ with $|A|=m^{\prime}$ we will show that there is $k \in \mathbb{Z}$ such that $\left|f^{k}(A)\right|_{\delta}>n^{\prime}$, i.e., the same expansive constant works. We divide the proof in two cases. 
First assume that $m^{\prime} \geq m$. Let $B \subset A$ with $|B|=m$. Since $f$ is $(m, n)$ expansive, there is $k \in \mathbb{Z}$ such that $\left|f^{k}(B)\right|_{\delta}>n$. Therefore $\left|f^{k}(A)\right|_{\delta}>n \geq n^{\prime}$, proving that $f$ is $\left(m^{\prime}, n^{\prime}\right)$-expansive.

Now suppose that $m^{\prime}<m$. Given that $|A|=m^{\prime}$ and $|X|=\infty$ there is $C \subset X$ such that $A \cap C=\emptyset$ and $|A \cup C|=m$. By $(m, n)$-expansiveness, there is $k \in \mathbb{Z}$ such that $\left|f^{k}(A \cup C)\right|_{\delta}>n$. Then, there is a $\delta$-separated set $D \subset f^{k}(A \cup C)$ with $|D|>n$. Notice that

$$
\left|f^{k}(A) \cap D\right|=\left|D \backslash f^{k}(C)\right| \geq|D|-\left|f^{k}(C)\right|>n-\left(m-m^{\prime}\right)
$$

and since $n-\left(m-m^{\prime}\right) \geq n^{\prime}$ by hypothesis, we have that $f^{k}(A) \cap D$ is a $\delta$ separated subset of $f^{k}(A)$ with more than $n^{\prime}$ points, that is $\left|f^{k}(A)\right|_{\delta}>n^{\prime}$. This proves the $\left(m^{\prime} n^{\prime}\right)$-expansiveness of $f$ in this case too.

As a consequence of Proposition 1.3 we have that

(1) $(m, n)$-expansive implies $(m+1, n)$-expansive and

(2) $(m, n)$-expansive implies $(m-1, n-1)$-expansive.

In Table 1 below we can easily see all these implications. The following proposition allows us to draw more arrows in this table, for example: $(4,2) \Rightarrow(2,1)$.

TABLE 1. Basic hierarchy of $(m, n)$-expansiveness. Each pair $(m, n)$ in the table stands for " $(m, n)$-expansive". In the first position, $(2,1)$, we have expansiveness. The first line, of the form $(N+1,1)$, we have $N$-expansive homeomorphisms.

$$
\begin{aligned}
& (2,1) \Rightarrow(3,1) \Rightarrow(4,1) \quad \Rightarrow \quad \ldots \\
& \begin{array}{c}
\Uparrow \\
(3,2)
\end{array} \Rightarrow \begin{array}{c}
\Uparrow \\
(4,2)
\end{array} \Rightarrow(5,2) \Rightarrow \ldots \\
& \underset{\Uparrow}{(3,2)} \underset{\Uparrow}{(} \rightarrow \underset{ }{(4,2)} \rightarrow \\
& (4,3) \Rightarrow(5,3) \Rightarrow(6,3) \Rightarrow \ldots \\
& \Uparrow \quad \Uparrow \quad \Uparrow
\end{aligned}
$$

Proposition 1.4. If $a, n \geq 2$ and $f: X \rightarrow X$ is an (an, $n$ )-expansive homeomorphism, then $f$ is $(a, 1)$-expansive.

In order to prove it, let us introduce two previous results.

Lemma 1.5. If $A, B \subset X$ are finite sets and $\delta>0$ satisfies $|A|=|A|_{\delta}$ and $|B|_{\delta}=1$, then for all $\varepsilon>0$ it holds that

$$
|A \cup B|_{\delta+\varepsilon} \leq|A|_{\varepsilon}+|B|_{\delta}-|A \cap B| .
$$

Proof. If $A \cap B=\emptyset$, then the proof is easy because

$$
|A \cup B|_{\delta+\varepsilon} \leq|A|_{\delta+\varepsilon}+|B|_{\delta+\varepsilon} \leq|A|_{\varepsilon}+|B|_{\delta} .
$$


Assume now that $A \cap B \neq \emptyset$. Since $|A|=|A|_{\delta}$ we have that $A$ is $\delta$-separated. Therefore $|A \cap B|=1$ because $|B|_{\delta}=1$. Assume that $A \cap B=\{y\}$. Let us prove that $|A \cup B|_{\delta+\varepsilon} \leq|A|_{\varepsilon}$ and notice that it is sufficient to conclude the proof of the lemma.

Let $C \subset A \cup B$ be a $(\delta+\varepsilon)$-separated set such that $|C|=|A \cup B|_{\delta+\varepsilon}$. If $C \subset A$, then

$$
|A \cup B|_{\delta+\varepsilon}=|A|_{\delta+\varepsilon} \leq|A|_{\varepsilon} .
$$

Therefore, let us assume that there is $x \in C \backslash A$. Define the set

$$
D=(C \cup\{y\}) \backslash\{x\} .
$$

Notice that $|C|=|D|$ and $D \subset A$.

We will show that $D$ is $\varepsilon$-separated. Take $p, q \in D$ and arguing by contradiction assume that $p \neq q$ and $\operatorname{dist}(p, q) \leq \varepsilon$. If $p, q \in C$ there is nothing to prove because $C$ is $(\delta+\varepsilon)$-separated. Assume now that $p=y$. We have that $\operatorname{dist}(x, p) \leq \delta$ because $x, p \in B$ and $|B|_{\delta}=1$. Thus

$$
\operatorname{dist}(x, q) \leq \operatorname{dist}(x, p)+\operatorname{dist}(p, q) \leq \varepsilon+\delta .
$$

But this is a contradiction because $x, q \in C$ and $C$ is $(\varepsilon+\delta)$-separated.

Lemma 1.6. If $f$ is $(m+l, n+1)$-expansive, then $f$ is $(m, n)$-expansive or $(l, 1)$-expansive.

Proof. Let us argue by contradiction and take an $(m+l, n+1)$-expansive constant $\alpha>0$. Since $f$ is not $(m, n)$-expansive for $\varepsilon \in(0, \alpha)$ there is a set $A \subset X$ such that $|A|=m$ and $\left|f^{k}(A)\right|_{\varepsilon} \leq n$ for all $k \in \mathbb{Z}$. Take $\delta>0$ such that $|A|=|A|_{\delta}$ and $\delta+\varepsilon<\alpha$.

Since $f$ is not $(l, 1)$-expansive there is $B$ such that $|B|=l$ and $\left|f^{k}(B)\right|_{\delta}=1$ for all $k \in \mathbb{Z}$. By Lemma 1.5 we have that

$$
\left|f^{k}(A \cup B)\right|_{\delta+\varepsilon} \leq\left|f^{k}(A)\right|_{\varepsilon}+\left|f^{k}(B)\right|_{\delta}-|A \cap B| \leq n+1-|A \cap B|
$$

for all $k \in \mathbb{Z}$. Also, we know that $|A \cup B|=m+l-|A \cap B|$. If we denote $r=|A \cap B|$, then $f$ is not $(m+l-r, n+1-r)$-expansive. And by Proposition 1.3 we conclude that $f$ is not $(m+l, n+1)$-expansive. This contradiction proves the lemma.

Proof of Proposition 1.4. Assume by contradiction that $f$ is not $(a, 1)$-expansive. Since $f$ is $(a n, n)$-expansive, by Lemma 1.6 we have that $f$ has to be $(a(n-1), n-1)$-expansive. Arguing inductively we can prove that $f$ is $(a(n-$ $j), n-j$ )-expansive for $j=1,2, \ldots, n-1$. In particular, $f$ is $(a, 1)$-expansive, which is a contradiction that proves the proposition.

Corollary 1.7. If $m \leq$ an and $f$ is $(m, n)$-expansive, then $f$ is $(a, 1)$-expansive (i.e., $(a-1)$-expansive in the sense of [9]). In particular, if $m \leq 2 n$ and $f$ is $(m, n)$-expansive, then $f$ is expansive.

Proof. By Proposition 1.3 we have that $f$ is $(a n, n)$-expansive. Therefore, by Proposition 1.4 we have that $f$ is $(a, 1)$-expansive. 


\section{Separating 4 points}

In this section we prove that $(n+1, n)$-expansiveness with $n \geq 3$ implies that $X$ is finite.

Theorem 2.1. If $X$ is a compact metric space admitting a $(4,3)$-expansive homeomorphism, then $X$ is a finite set.

Proof. By contradiction assume that $f$ is a $(4,3)$-expansive homeomorphism of $X$ with $|X|=\infty$ and take an expansive constant $\delta>0$. We know that $f$ cannot be positive expansive (see $[4,7]$ for a proof). Therefore there are $x_{1}, x_{2}$ such that $x_{1} \neq x_{2}$ and

$$
\operatorname{dist}\left(f^{k}\left(x_{1}\right), f^{k}\left(x_{2}\right)\right)<\delta
$$

for all $k \geq 0$. Analogously, $f^{-1}$ is not positive expansive, and we can take $y_{1}, y_{2}$ such that $y_{1} \neq y_{2}$ and

$$
\operatorname{dist}\left(f^{k}\left(y_{1}\right), f^{k}\left(y_{2}\right)\right)<\delta
$$

for all $k \leq 0$. Consider the set $A=\left\{x_{1}, x_{2}, y_{1}, y_{2}\right\}$. We have that $2 \leq|A| \leq 4$ (we do not know if the 4 points are different). By inequalities (1) and (2) we have that $\left|f^{k}(A)\right|_{\delta}<|A|$ for all $k \in \mathbb{Z}$. If $n=|A|$, then we have that $f$ is not $(n, n-1)$-expansive. In any case, $n=2,3$ or 4 , by Proposition 1.3 (see Table 1 ) we conclude that $f$ is not $(4,3)$-expansive. This contradiction finishes the proof.

Remark 2.2. If $X$ is a compact metric space admitting a $(n+1, n)$-expansive homeomorphism with $n \geq 3$, then $X$ is a finite set. It follows by Theorem 2.1 and Proposition 1.3.

Corollary 2.3. If $f: X \rightarrow X$ is a homeomorphism of a compact metric space and $|X|=\infty$, then for all $\delta>0$ and $m \geq 4$ there is $A \subset X$ with $|A|=m$ such that $\left|f^{k}(A)\right|_{\delta}<|A|$ for all $k \in \mathbb{Z}$.

Proof. It is just a restatement of Remark 2.2.

\section{On Peano continua}

In this section we study $(m, n)$-expansiveness on Peano continua. Let us start recalling that a continuum is a compact connected metric space and a Peano continuum is a locally connected continuum. A singleton space $(|X|=1)$ is a trivial Peano continuum. For $x \in X$ and $\delta>0$ define the stable and unstable set of $x$ as

$$
\begin{aligned}
& W_{\delta}^{s}(x)=\left\{y \in X: \operatorname{dist}\left(f^{k}(x), f^{k}(y)\right) \leq \delta \forall k \geq 0\right\}, \\
& W_{\delta}^{u}(x)=\left\{y \in X: \operatorname{dist}\left(f^{k}(x), f^{k}(y)\right) \leq \delta \forall k \leq 0\right\} .
\end{aligned}
$$

Remark 3.1. Notice that $(m, n)$-expansiveness implies continuum-wise expansiveness for all $m>n \geq 1$. Recall that $f$ is continuum-wise expansive if there is $\delta>0$ such that if $\operatorname{diam}\left(f^{k}(A)\right)<\delta$ for all $k \in \mathbb{Z}$ and some continuum $A \subset X$, then $|A|=1$. 
Theorem 3.2. If $X$ is a non-trivial Peano continuum, then no homeomorphism of $X$ is $(m, n)$-expansive if $2 m \geq 3 n$.

Proof. Let $\delta$ be a positive real number and assume that $f$ is $(m, n)$-expansive. As we remarked above, $f$ is a continuum-wise expansive homeomorphism. It is known (see $[5,6]$ ) that for such homeomorphisms on a Peano continuum, every point has non-trivial stable and unstable sets. Take $n$ different points $x_{1}, \ldots, x_{n} \in X$ and let $\delta^{\prime} \in(0, \delta)$ be such that $\operatorname{dist}\left(x_{i}, x_{j}\right)>2 \delta^{\prime}$ if $i \neq j$. For each $i=1, \ldots, n$, we can take $y_{i} \in W_{\delta^{\prime}}^{s}\left(x_{i}\right)$ and $z_{i} \in W_{\delta^{\prime}}^{u}\left(x_{i}\right)$ with $x_{i} \neq y_{i}$ and $x_{i} \neq z_{i}$. Consider the set $A=\left\{x_{1}, y_{1}, z_{1}, \ldots, x_{n}, y_{n}, z_{n}\right\}$. Since $\operatorname{dist}\left(x_{i}, x_{j}\right)>$ $2 \delta^{\prime}$ if $i \neq j$, and $y_{i}, z_{i} \in B_{\delta^{\prime}}\left(x_{i}\right)$ we have that $|A|=3 n$. If $A_{i}$ denotes the set $\left\{x_{i}, y_{i}, z_{i}\right\}$ we have that $\left|f^{k}\left(A_{i}\right)\right|_{\delta^{\prime}} \leq 2$ for all $k \in \mathbb{Z}$. This is because if $k \geq 0$, then $\operatorname{dist}\left(f^{k}\left(x_{i}\right), f^{k}\left(y_{i}\right)\right) \leq \delta^{\prime}$ and if $k \leq 0$, then $\operatorname{dist}\left(f^{k}\left(x_{i}\right), f^{k}\left(z_{i}\right)\right) \leq \delta^{\prime}$. Therefore $\left|f^{k}(A)\right|_{\delta^{\prime}} \leq 2 n$, and then $\left|f^{k}(A)\right|_{\delta} \leq 2 n$. Since $\delta>0$ and $n \geq 1$ are arbitrary, we have that $f$ is not $(3 n, 2 n)$ expansive for all $n \geq 1$. Finally, by Proposition 1.3, we have that $f$ is not $(m, n)$-expansive if $2 m \geq 3 n$.

Corollary 3.3. If $f: X \rightarrow X$ is a homeomorphism and $X$ is a non-trivial Peano continuum, then for all $\delta>0$ there is $A \subset X$ such that $|A|=3$ and $\left|f^{k}(A)\right|_{\delta} \leq 2$ for all $k \in \mathbb{Z}$.

Proof. By Theorem 3.2 we know that $f$ is not $(3,2)$-expansive. Therefore, the proof follows by definition.

\section{Hyper-expansive homeomorphisms}

Denote by $\mathcal{K}(X)$ the set of compact subsets of $X$. This space is usually called as the hyper-space of $X$. We recommend the reader to see [11] for more on the subject of hyper-spaces and the proofs of the results that we will cite below. In the set $\mathcal{K}(X)$ we consider the Hausdorff distance $\operatorname{dist}_{H}$ making $\left(\mathcal{K}(X), \operatorname{dist}_{H}\right)$ a compact metric space. Recall that

$$
\operatorname{dist}_{H}(A, B)=\inf \left\{\varepsilon>0: A \subset B_{\varepsilon}(B) \text { and } B \subset B_{\varepsilon}(A)\right\},
$$

where $B_{\varepsilon}(C)=\cup_{x \in C} B_{\varepsilon}(x)$ and $B_{\varepsilon}(x)$ is the usual ball of radius $\varepsilon$ centered at $x$. As usual, we let $f$ to act on $\mathcal{K}(X)$ as $f(A)=\{f(a): a \in A\}$.

Definition 3. We say that $f$ is hyper-expansive if $f: \mathcal{K}(X) \rightarrow \mathcal{K}(X)$ is expansive, i.e., there is $\delta>0$ such that given two compact sets $A, B \subset X, A \neq B$, there is $k \in \mathbb{Z}$ such that $\operatorname{dist}_{H}\left(f^{k}(A), f^{k}(B)\right)>\delta$ where $\operatorname{dist}_{H}$ is the Hausdorff distance.

In [1] it is shown that $f: X \rightarrow X$ is hyper-expansive if and only if $f$ has a finite number of orbits (i.e., there is a finite set $A \subset X$ such that $X=$ $\left.\cup_{k \in \mathbb{Z}} f^{k}(A)\right)$ and the non-wandering set is a finite union of periodic points which are attractors or repellers. Recall that a point $x$ is in the non-wandering set if for every neighborhood $U$ of $x$ there is $k>0$ such that $f^{k}(U) \cap U \neq \emptyset$. A point $x$ is periodic if for some $k \geq 0$ it holds that $f^{k}(x)=x$. The orbit 
$\gamma=\left\{x, f(x), \ldots, f^{k-1}(x)\right\}$ is a periodic orbit if $x$ is a periodic point. A periodic orbit $\gamma$ is an attractor (repeller) if there is a compact neighborhood $U$ of $\gamma$ such that $f^{k}(U) \rightarrow \gamma$ in the Hausdorff distance as $k \rightarrow \infty$ (resp. $k \rightarrow-\infty$ ).

Theorem 4.1. If $f: X \rightarrow X$ is a hyper-expansive homeomorphism and $|X|=$ $\infty$, then $f$ is $(m, n)$-expansive for some $m>n \geq 1$ if and only if $m \leq 3$.

Proof. Let us start with the direct part of the theorem. Let $P_{a}$ be the set of periodic attractors, $P_{r}$ the set of periodic repellers and take $x_{1}, \ldots, x_{j}$ one point in each wandering orbit. (Recall that, as we said above, hyper-expansiveness implies that $f$ has just a finite number of orbits.) Define $Q=\left\{x_{1}, \ldots, x_{j}\right\}$. Take $\delta>0$ such that

(1) if $p, q \in P_{a} \cup P_{r}$ and $p \neq q$, then $\operatorname{dist}(p, q)>\delta$,

(2) if $x_{i} \in Q$, then $B_{\delta}\left(x_{i}\right)=\left\{x_{i}\right\}$ (recall that wandering points are isolated by [1]),

(3) if $p \in P_{a}, x_{i} \in Q$ and $k \leq 0$, then $\operatorname{dist}\left(p, f^{k}\left(x_{i}\right)\right)>\delta$,

(4) if $q \in P_{r}, x_{i} \in Q$ and $k \geq 0$, then $\operatorname{dist}\left(p, f^{k}\left(x_{i}\right)\right)>\delta$ and

(5) if $x, y \in Q$ and $k>0>l$, then $\operatorname{dist}\left(f^{k}(x), f^{l}(y)\right)>\delta$.

Let us prove that such $\delta$ is a $(3,2)$-expansive constant. Take $a, b, c \in X$ with $|\{a, b, c\}|=3$. The proof is divided by cases:

- If $a, b, c \in P=P_{a} \cup P_{r}$, then item 1 above concludes the proof.

- If $a, b \in P$ and $c \notin P$, then there is $k \in \mathbb{Z}$ such that $f^{k}(c) \in Q$. In this case items 1 and 2 conclude the proof.

- Assume now that $a \in P$ and $b, c \notin P$. Without loss of generality let us suppose that $a$ is a repeller. Let $k_{b}, k_{c} \in \mathbb{Z}$ be such that $f^{k_{b}}(b), f^{k_{c}}(c) \in$ $Q$. Define $k=\min \left\{k_{b}, k_{c}\right\}$. In this way: $\operatorname{dist}\left(f^{k}(a), f^{k}(b)\right), \operatorname{dist}\left(f^{k}(a)\right.$, $\left.f^{k}(c)\right) \geq \delta$ by item 4 and $\operatorname{dist}\left(f^{k}(b), f^{k}(c)\right) \geq \delta$ by item 2 .

- If $a, b, c \notin P$, then consider $k_{a}, k_{b}, k_{c} \in \mathbb{Z}$ such that $f^{k_{a}}(a), f^{k_{b}}(b), f^{k_{c}}(c)$ $\in Q$. Assume, without loss, that $k_{a} \leq k_{b} \leq k_{c}$. Take $k=k_{b}$. In this way, items 2 and 5 finishes the direct part of the proof.

To prove the converse, we will show that $f$ is not $(m, 3)$-expansive for all $m>$ 3. Take $\delta>0$. Notice that since $X=\infty$ there is at least one wandering point $x$. Without loss of generality assume that $\lim _{k \rightarrow \infty} f^{k}(x)=p_{a}$ an attractor fixed point and $\lim _{k \rightarrow-\infty} f^{k}(x)=p_{r}$ a repeller fixed point. Take $k_{1}, k_{2} \in \mathbb{Z}$ such that $\operatorname{dist}\left(f^{k}(x), p_{r}\right)<\delta$ for all $k \leq k_{1}$ and $\operatorname{dist}\left(f^{k}(x), p_{a}\right)<\delta$ for all $k \geq k_{2}$. Let $l=k_{2}-k_{1}$ and define $x_{1}=f^{-k_{1}}(x)$, and $x_{i+1}=f^{l}\left(x_{i}\right)$ for all $i \geq 1$. Consider the set $A=\left\{x_{1}, \ldots, x_{m}\right\}$. By construction we have that $|A|=m$ and $\left|f^{k}(A)\right|_{\delta} \leq 3$ for all $k \in \mathbb{Z}$. Thus, proving that $f$ is not $(m, 3)$-expansive if $m>3$.

Remark 4.2. In light of the previous proof one may wonder if a smart $\delta$-observer will not be able to say that $A$ has more than 3 points. We mean, we are assuming that a $\delta$-observer will say that $A$ has $n^{\prime}$ points with

$$
n^{\prime}=\max _{k \in \mathbb{Z}}\left|f^{k}(A)\right|_{\delta}
$$


According to the dynamic of the set $A$ in the previous proof, we guess that with more reasoning a smarter $\delta$-observer will find that $A$ has more than 3 points.

Theorem 4.1 gives us examples of $(3,2)$-expansive homeomorphisms on infinite countable compact metric spaces. A natural question is: does $(3,2)$ expansiveness implies hyper-expansiveness? I do not know the answer, but let us remark some facts that may be of interest. If $f$ is $(3,2)$-expansive, then:

- For all $x \in X$ either the stable or the unstable set must be trivial. It follows by the arguments of the proof of Theorem 3.2.

- If $x, y$ are doubly asymptotic, i.e., $\operatorname{dist}\left(f^{k}(x), f^{k}(y)\right) \rightarrow 0$ as $k \rightarrow \pm \infty$, then they are isolated points of the space. Suppose that $x$ were an accumulation point. Given $\delta>0$ take $k_{0}$ such that if $|k|>k_{0}$, then $\operatorname{dist}\left(f^{k}(x), f^{k}(y)\right)<\delta$. Take a point $z$ close to $x$ such that $\operatorname{dist}\left(f^{k}(x)\right.$, $\left.f^{k}(z)\right)<\delta$ if $|k| \leq k_{0}$ (we are just using the continuity of $f$ ). Then $x, y, z$ contradicts $(3,2)$-expansiveness.

Proposition 4.3. There are $(4,2)$-expansive homeomorphisms that are not $(3,2)$-expansive.

Proof. Let us prove it giving an example. Consider a countable compact metric space $X$ and a homeomorphism $f: X \rightarrow X$ with the following properties:

(1) $f$ has 5 orbits,

(2) $a, b, c \in X$ are fixed points of $f$,

(3) there is $x \in X$ such that $\lim _{k \rightarrow-\infty} f^{k}(x)=a$ and $\lim _{k \rightarrow+\infty} f^{k}(x)=b$,

(4) there is $y \in X$ such that $\lim _{k \rightarrow-\infty} f^{k}(y)=b$ and $\lim _{k \rightarrow+\infty} f^{k}(y)=c$.

In order to see that $f$ is not $(3,2)$-expansive consider $\varepsilon>0$. Take $k_{0} \in \mathbb{Z}$ such that for all $k \geq k_{0}$ it holds that $\operatorname{dist}\left(f^{k}(x), b\right)<\varepsilon$ and $\operatorname{dist}\left(f^{-k}(y), b\right)<\varepsilon$. Define $u=f^{k_{0}}(x)$ and $v=f^{-k_{0}}(y)$. In this way $\left\|\left\{f^{k}(u), b, f^{k}(v)\right\}\right\|_{\varepsilon} \leq 2$ for all $k \in \mathbb{Z}$. This proves that $f$ is not $(3,2)$-expansive.

Let us now indicate how to prove that $f$ is $(4,2)$-expansive. Consider $\varepsilon>0$ such that if $i \geq 0$ and $j \in \mathbb{Z}$, then $\operatorname{dist}\left(f^{-i}(x), f^{j}(y)\right)>\varepsilon$ and $\operatorname{dist}\left(f^{j}(x), f^{i}(y)\right)$ $>\varepsilon$. Now, a similar argument as in the proof of Theorem 4.1, shows that $f$ is $(4,2)$-expansive.

\section{With the shadowing property}

In this section we prove that an important class of homeomorphisms are not $(m, n)$-expansive for all $m>n \geq 2$. In order to state this result let us recall that a $\delta$-pseudo orbit is a sequence $\left\{x_{k}\right\}_{k \in \mathbb{Z}}$ such that $\operatorname{dist}\left(f\left(x_{k}\right), x_{k+1}\right) \leq \delta$ for all $k \in \mathbb{Z}$. We say that a homeomorphism has the shadowing property if for all $\varepsilon>0$ there is $\delta>0$ such that if $\left\{x_{k}\right\}_{k \in \mathbb{Z}}$ is a $\delta$-pseudo orbit, then there is $x$ such that $\operatorname{dist}\left(f^{k}(x), x_{k}\right)<\varepsilon$ for all $k \in \mathbb{Z}$. In this case we say that $x \varepsilon$-shadows the $\delta$-pseudo orbit. 
Theorem 5.1. Let $f: X \rightarrow X$ be a homeomorphism of a compact metric space $X$. If $f$ has the shadowing property and there are $x, y \in X$ such that

$$
0=\liminf _{k \rightarrow \infty} \operatorname{dist}\left(f^{k}(x), f^{k}(y)\right)<\limsup _{k \rightarrow \infty} \operatorname{dist}\left(f^{k}(x), f^{k}(y)\right),
$$

then $f$ is not $(m, n)$-expansive if $m>n \geq 2$.

Proof. By Proposition 1.3 it is enough to prove that $f$ cannot be $(m, 2)$ expansive if $m>2$. Consider $\varepsilon>0$. We will define a set $A$ with $|A|=\infty$ such that for all $k \in \mathbb{Z}, f^{k}(A) \subset B_{\varepsilon}\left(f^{k}(x)\right) \cup B_{\varepsilon}\left(f^{k}(y)\right)$, proving that $f$ is not $(m, 2)$-expansive for all $m>2$.

Consider two increasing sequences $a_{l}, b_{l} \in \mathbb{Z}, \rho \in(0, \varepsilon)$ and $\delta>0$ such that

$$
\begin{aligned}
& a_{1}<b_{1}<a_{2}<b_{2}<a_{3}<b_{3}<\cdots, \\
& \operatorname{dist}\left(f^{a_{l}}(x), f^{a_{l}}(y)\right)<\delta \\
& \operatorname{dist}\left(f^{b_{l}}(x), f^{b_{l}}(y)\right)>\rho
\end{aligned}
$$

for all $l \geq 1$ and assume that every $\delta$-pseudo orbit can be $(\rho / 2)$-shadowed. For each $l \geq 1$ define the $\delta$-pseudo orbit $z_{k}^{l}$ as

$$
z_{k}^{l}=\left\{\begin{array}{l}
f^{k}(x) \text { if } k<a_{l}, \\
f^{k}(y) \text { if } k \geq a_{l} .
\end{array}\right.
$$

Then, for every $l \geq 1$ there is a point $w^{l}$ whose orbit $(\rho / 2)$-shadows the $\delta$ pseudo orbit $\left\{z_{k}^{l}\right\}_{k \in \mathbb{Z}}$. Let us now prove that if $1 \leq l<s$, then $w^{l} \neq w^{s}$. We have that $a_{l}<b_{l}<a_{s}$. Therefore $z_{b_{l}}^{l}=f^{b_{l}}(y)$ and $z_{b_{l}}^{s}=f^{b_{l}}(x)$. Since $w^{l}$ and $w^{s}(\rho / 2)$-shadows the pseudo orbits $z^{l}$ and $z^{s}$, respectively, we have that

$$
\operatorname{dist}\left(f^{b_{l}}\left(w^{l}\right), f^{b_{l}}(y)\right), \operatorname{dist}\left(f^{b_{l}}\left(w^{s}\right), f^{b_{l}}(x)\right)<\rho / 2 .
$$

We conclude that $w^{l} \neq w^{s}$ because $\operatorname{dist}\left(f^{b_{l}}(x), f^{b_{l}}(y)\right)>\rho$. Therefore, if we define $A=\left\{w^{l}: l \geq 1\right\}$ we have that $|A|=\infty$. Finally, since $\rho<\varepsilon$, we have that $f^{k}(A) \subset B_{\varepsilon}\left(f^{k}(x)\right) \cup B_{\varepsilon}\left(f^{k}(y)\right)$ for all $k \in \mathbb{Z}$. Therefore, $\left|f^{k}(A)\right|_{\varepsilon} \leq 2$ for all $k \in \mathbb{Z}$.

Remark 5.2. Examples where Theorem 5.1 can be applied are Anosov diffeomorphisms and symbolic shift maps. Also, if $f: X \rightarrow X$ is a homeomorphism with an invariant set $K \subset X$ such that $f: K \rightarrow K$ is conjugated to a symbolic shift map, then Theorem 5.1 holds because the $(m, n)$-expansiveness of $f$ in $X$ implies the $(m, n)$-expansiveness of $f$ restricted to any compact invariant set $K \subset X$ as can be easily checked.

\section{References}

[1] A. Artigue, Hyper-expansive homeomorphisms, Publ. Mat. Urug. 14 (2013), 62-66.

[2] A. Artigue, M. J. Pacifico, and J. L. Vieitez, $N$-expansive homeomorphisms on surfaces, Preprint, 2013.

[3] R. Bowen, Entropy-expansive maps, Trans. Amer. Math. Soc. 164 (1972), 323-331.

[4] E. M. Coven and M. Keane, Every compact metric space that supports a positively expansive homeomorphism is finite, IMS Lecture Notes Monogr. Ser., Dynamics \& Stochastics 4 (2006), 304-305. 
[5] J. R. Hertz, There are no stable points for continuum-wise expansive homeomorphisms, Pre. Mat. Urug. 65 (2002).

[6] H. Kato, Continuum-wise expansive homeomorphisms, Canad. J. Math. 45 (1993), no. $3,576-598$

[7] J. Lewowicz, Dinámica de los homeomorfismos expansivos, Monografías del IMCA, 2003.

[8] C. A. Morales, Measure expansive systems, Preprint IMPA, 2011.

[9] — A generalization of expansivity, Discrete Contin. Dyn. Syst. 32 (2012), no. 1, 293-301.

[10] C. A. Morales and V. F. Sirvent, Expansive Measures, IMPA, 29o Colóq. Bras. Mat., 2013.

[11] S. Nadler Jr., Hyperspaces of Sets, Marcel Dekker Inc. New York and Basel, 1978.

[12] M. J. Pacifico and J. L. Vieitez, Entropy expansiveness and domination for surface diffeomorphisms, Rev. Mat. Complut. 21 (2008), no. 2, 293-317.

[13] W. L. Reddy, Pointwise expansion homeomorphisms, J. Lond. Math. Soc. 2 (1970), $232-236$.

[14] K. Sakai, Continuum-wise expansive diffeomorphisms, Publ. Mat. 41 (1997), no. 2, 375382 .

[15] S. Schwartzman, On transformation groups, Dissertation, Yale University, 1952.

[16] W. R. Utz, Unstable homeomorphisms, Proc. Amer. Math. Soc. 1 (1950), no. 6, 769-774.

Departamento de Matemática y Estadística del Litoral

UNIVERSIDAD DE LA REPÚBLICA

Gral. Rivera 1350, Salto, Uruguay

E-mail address: artigueQunorte.edu.uy 